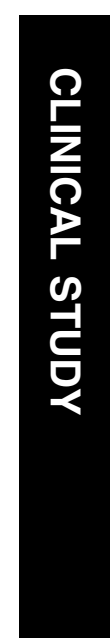

\section{The incidence of endophthalmitis following transconjunctival sutureless 25- vs 20-gauge vitrectomy}

${ }^{1}$ The Johns Hopkins University School of Medicine, Baltimore, MD, USA

${ }^{2}$ Retina Division, Wilmer Eye Institute, The Johns Hopkins University School of Medicine, Baltimore, MD, USA

Correspondence: DV Do, Wilmer Eye Institute, The Johns Hopkins University School of Medicine,

600 North Wolfe Street, Maumenee 740, Baltimore, MD 21287, USA

Tel: + 1410955 3518;

Fax: + 14109553500

E-mail: ddo@jhmi.edu

Received: 30 January 2008 Accepted in revised form: 25 April 2008

Published online: 6 June 2008

We thank the faculty members of the Wilmer Eye Institute Retina Division (Peter A Campochiaro, Julia A Haller, James T Handa, Peter Gehlbach, Sharon Solomon, Jennifer U Sung, Howard Ying, and Ingrid Zimmer-Galler).

The authors have no financial conflicts of interest.
Abstract

Aim To determine the incidence of endophthalmitis following 25-gauge and standard 20-gauge vitrectomy.

Methods In this single-centre retrospective interventional case series, we evaluated the incidence of acute endophthalmitis occurring within the 14-day postoperative period in all patients who underwent pars plana vitrectomy between 1 November 2002 and 31 December 2006. A total of 3477 consecutive patients were identified. Of them, 3046 patients underwent 20 -gauge vitrectomy and 431 underwent 25-gauge vitrectomy.

Results The event rates of postoperative endophthalmitis were $0.03 \%$ ( 1 of 3046) after 20 -gauge vitrectomy and $0.23 \%$ ( 1 of 431$)$ after 25-gauge vitrectomy $(P=0.23)$. In the endophthalmitis case that occurred after 25-gauge vitrectomy, a combined phacoemulsification cataract surgery was performed with the 25-gauge vitrectomy. Analyses of event rates of postoperative endophthalmitis after combining phacoemulsification cataract surgery and vitrectomy were $0 \%(0$ of 170$)$ for 20 gauge vitrectomy and $2.17 \%$ (1 of 46 ) for 25 -gauge surgery vitrectomy $(P=0.21)$.

Conclusion The incidence of postoperative endophthalmitis following 25-gauge vitrectomy and 20-gauge surgery is low. Although there is a trend suggesting an increased risk of endophthalmitis with the 25-gauge system, this difference was not statistically significant given the low number of measured outcomes.

Eye (2009) 23, 780-784; doi:10.1038/eye.2008.160; published online 6 June 2008

Keywords: vitrectomy; endophthalmitis;

20-guage; 25-guage
JK Chen ${ }^{1}$, RN Khurana ${ }^{2}$, QD Nguyen ${ }^{2}$ and DV Do ${ }^{2}$

Introduction

The 25-gauge transconjunctival sutureless vitrectomy system was first introduced as an alternative to the traditional 20-gauge system by Fujii et al in 2003. ${ }^{1}$ Theoretical benefits of the 25-gauge system include decreased iatrogenic trauma to the eye at entry sites, elimination of conjunctival peritomy, reduction of postoperative astigmatism, and decreased operative time. ${ }^{1,2}$ In addition, 25-gauge vitrectomy has been reported to hasten postoperative recovery, as the smaller, selfsealing surgical openings facilitate wound healing and minimize pain, while possibly reducing postoperative inflammation. ${ }^{2-7}$ Reported complications include retinal break, retinal detachment, macular hole, cataract progression, hypotony, and breakage of the 25-gauge cutter within the eye. ${ }^{2,8-10}$ More concerning, several reports have suggested that the 25-gauge system may result in an increased incidence of postoperative endophthalmitis, ${ }^{11-15}$ which can lead to significant morbidity as potential sequelae include irreversible loss of vision. The purpose of this study was to estimate the incidence of endophthalmitis after sutureless 25-gauge vitrectomy surgery and to determine whether there was an increased risk of infection after 25-gauge vitrectomy compared to traditional 20-gauge vitrectomy.

Materials and methods

This was a single-centre, retrospective, interventional case series in which we conducted retrospective chart review of 3477 consecutive patients who underwent pars plana vitrectomy (PPV) at Wilmer Eye Institute between 1 November 2002, when 25-gauge 
vitrectomy was first performed at this institution, and 31 December 2006, to identify cases of endophthalmitis after 25- and 20-gauge vitrectomy. All surgeries were performed by Wilmer Retina Division vitreoretinal surgeons. Patients who underwent combined scleral buckle and vitrectomy surgery were excluded, as were patients who underwent 23-gauge vitrectomy as that technique was not widely used in this hospital until the year 2007. The primary outcome was the incidence of acute endophthalmitis diagnosed within 14 days after surgery as a postoperative complication. The study protocol was approved by the Institutional Review Board of Johns Hopkins Medical Institutions.

Vitrectomy cases were identified through surgical billing records and cross-referenced with operating room purchasing orders to confirm all cases of 25- and 20-gauge vitrectomy performed at this hospital. Endophthalmitis cases were identified through ICD-9 billing codes. Ophthalmology records of endophthalmitis cases were reviewed to determine operative surgical techniques, postoperative course, and culture results. Significance between endophthalmitis rates was determined using a Fisher's exact test. A $P$-value less than 0.05 was considered statistically significant.

A standard eye preparation was used in all surgical cases: $5 \%$ povidone iodine was used to clean the eyelid margins, eyelashes, and irrigate the conjunctiva; sterile surgical drapes and lid speculum were used in a standard operative fashion; infusion fluid consisted of balanced saline solution without antibiotics; and subconjunctival injections of antibiotics at the conclusion of surgery were at the discretion of the surgeon. Postoperative antibiotic eyedrops were routinely used for 7 days following surgery. No preoperative antibiotics were used.

\section{Results}

There were 3477 cases of vitrectomy performed at the Wilmer Eye Institute from 1 November 2002 to 31 December 2006. There were 3046 cases of 20-gauge and 431 cases of 25-gauge vitrectomy. Among all the vitrectomy cases, 170 cases consisted of combination surgery of 20-gauge vitrectomy with phacoemulsification cataract surgery, and 46 cases consisted of combination surgery of 25-gauge vitrectomy with phacoemulsification cataract surgery.

There were two cases of acute postoperative endophthalmitis. One case involved 20-gauge surgery and the other, combination surgery of 25-gauge vitrectomy and phacoemulsification cataract extraction with intraocular (IOL) placement. The event rates of postoperative endophthalmitis were $0.03 \%$ ( 1 of 3046) after 20-gauge surgery and $0.23 \%$ (1 of 431) after 25-gauge surgery $(P=0.23)$. Event rates of postoperative endophthalmitis after combination vitrectomy with cataract extraction were $0 \%$ (0 of 170 ) for 20-gauge vitrectomy and $2.17 \%$ (1 of 46) for 25-gauge vitrectomy $(P=0.21)$. A detailed review of each endophthalmitis case is presented below.

\section{Case 1}

A 52-year-old diabetic woman with a non-clearing vitreous haemorrhage and visually significant cataract underwent combined 25-gauge vitrectomy with phacoemulsification cataract surgery to the left eye. Preoperative visual acuity was 20/200 OS. The patient underwent a clear corneal phacoemulsification and IOL implantation without complication. Two 10-0 nylon sutures were placed in an interrupted fashion across the clear corneal incision. For the 25-gauge trocar and cannula placement, the conjunctiva was not displaced and a straight incision involving the trocars was performed. A 25-gauge vitrectomy and endolaser scatter photocoagulation were performed without complication. At the conclusion of the surgery, the sclerotomy wounds, which were not sutured, and the sutured cornea incision did not leak. Subconjunctival decadron $(4.0 \mathrm{mg} / \mathrm{ml})$ was injected in the inferior fornix without any effect on sclerotomy sites. On postoperative day 1, the retina was attached, intraocular pressure (IOP) was 12, and no signs of hypotony were noted. The patient was prescribed ofloxacin and prednisolone acetate four times daily.

On postoperative day 7 , the patient presented with eye pain and decreased vision. Visual acuity in the left eye decreased to hand motions. The anterior chamber had $4+$ cell and a $1 \mathrm{~mm}$ hypopyon. The patient underwent 20-gauge vitrectomy, anterior chamber washout, and intravitreal injection of amikacin $(0.4 \mathrm{mg}$ per $0.1 \mathrm{ml})$ and vancomycin $(0.1 \mathrm{mg}$ per $0.1 \mathrm{ml})$ for presumptive endophthalmitis. Postoperatively, the patient was placed on fortified vancomycin, ciprofloxacin, and prednisolone acetate every $2 \mathrm{~h}$, and homatropine two times daily. Vitreous cultures were negative and revealed mononuclear inflammatory cells. Visual acuity improved to $20 / 125$, and the vitreous cavity cleared to reveal a subretinal haemorrhage in the macula. After 1 month, the patient developed a rhegmatogenous retinal detachment in the operated eye. She underwent 20-gauge vitrectomy and scleral buckle placement, eventually recovering light perception at postoperative month 4 .

\section{Case 2}

A 75-year-old diabetic woman underwent a 20-gauge vitrectomy for a tractional retinal detachment and vitreous haemorrhage of the left eye. Preoperatively, visual acuity was 20/200 and the patient was 
pseudophakic. The patient underwent uncomplicated 20-gauge PPV, membrane peel, endolaser, and intravitreal injection of triamcinolone $(4 \mathrm{mg})$. On postoperative day 1 , the visual acuity was $20 / 400$ and IOP was $16 \mathrm{~mm} \mathrm{Hg}$. Topical moxifloxacin and prednisolone acetate four times daily were prescribed postoperatively.

On postoperative day 8 , the patient presented with pain and decreased vision in the operated eye. Visual acuity decreased to hand motions. There was $2+$ conjunctival injection, a corneal epithelial defect, and $3+$ cells in the anterior chamber with a $1 \mathrm{~mm}$ hypopyon. IOP of the left eye was $18 \mathrm{~mm} \mathrm{Hg}$. A vitreous tap and intravitreal injection of vancomycin $(1 \mathrm{mg}$ per $0.1 \mathrm{ml})$ and clindamycin ( $1 \mathrm{mg}$ per $0.1 \mathrm{ml}$ ) was performed. She was started on moxifloxacin hourly, and tapered on predforte to every other day. Vitreous culture was positive for Staphylococcus aureus sensitive to vancomycin and clindamycin. Pain resolved the following day, and the hypopyon and corneal epithelial defect resolved within the week. On postoperative month 19, visual acuity returned to $20 / 200$.

\section{Conclusion}

The risk of endophthalmitis after 20-gauge vitrectomy is rare, with an estimated incidence ranging from 0.039 to $0.07 \% \%^{12,16}$ however, studies have suggested that the incidence of endophthalmitis is increased in 25-gauge vitrectomy. Proposed aetiologies include that nonsutured wounds may allow entry of extraocular fluids and organisms. Hypotony, observed after 25-gauge vitrectomy with an incidence of 3.8-20\%, may serve as conduit for infection. ${ }^{2}$ For instance, in human cadaveric eyes, fluctuations of IOP following sutureless clear cornea surgery may allow entry of surface fluid into the anterior chamber during the initial postoperative period when the wound has not healed. ${ }^{17}$ Furthermore, with the 25-gauge vitrectomy system, the infusion flow rate is reduced sixfold, ${ }^{1}$ potentially decreasing the amount of fluid that dilutes or flushes out organisms within the eye.

In this study, we retrospectively reviewed 3477 consecutive vitrectomy cases and found that endophthalmitis occurred in $0.03 \%$ (1 of 3046) of 20 -gauge surgery, and in $0.23 \%$ ( 1 of 431 ) of 25 -gauge surgery. There have been several reports of endophthalmitis following 25-gauge transconjunctival sutureless vitrectomy. ${ }^{11,13-15}$ In addition, the first systematic study to document the incidence of endophthalmitis following 25-gauge vitrectomy was recently published. Kunimoto and Kaiser ${ }^{18}$ retrospectively analysed 8601 consecutive cases of vitrectomy and found that 25-gauge vitrectomy had a statistically significant 12 -fold higher incidence of endophthalmitis (culture results not recorded) compared with 20-gauge vitrectomy ( 0.23 vs $0.018 \%, P=0.004)$. All patients who developed endophthalmitis had fluid-filled vitreous cavity at the end of surgery (no air, gas, or silicone oil tamponade). The authors postulated that a fluid-filled cavity may compromise wound integrity compared with air or gas fill. In addition, intravitreal triamcinolone acetonide was used in $38 \%$ of endophthalmitis cases, but it is unclear if the use of intravitreal steroids is associated with increased risk of endophthalmitis when combined with sutureless surgery.

In another study, Scott $e t a l^{15}$ recently retrospectively examined 6375 patients who underwent 20-gauge PPV and 1307 patients who underwent 25-gauge PPV. The incidence of endophthalmitis was $0.03 \%$ for 20-gauge PPV compared to $0.84 \%$ for 25 -gauge PPV $(P<0.0001)$, which represents a 24 times increased risk with the sutureless 25 -gauge system. In the cases of endophthalmitis associated with the 25-gauge system, sclerotomy ports were created with straight incisions in $73 \%$ and angled incisions in $27 \%$ of cases. In addition, an adjuvant agent was used intraoperatively in three eyes (triamcinolone acetonide in two eyes, Trypan blue in one eye). All of the eyes were fluid filled at the end of surgery. Cultures were positive in seven cases, among which six cultures were identified as coagulase-negative staphylococci.

In a smaller study, Shaikh et $a l^{19}$ retrospectively examined the incidence of complications following 129 consecutive cases of 25-gauge vitrectomy and 129 consecutive cases of 20-gauge vitrectomy. Two cases of endophthalmitis (one culture positive) were reported (1.6\%) following 25-gauge vitrectomy and none following 20-gauge vitrectomy. The incidence of endophthalmitis (1.6\%) was eight times higher than what Kunimoto and Kaiser ${ }^{18}$ and the current study reported in 25-gauge vitrectomy and two times larger than what Scott reported. ${ }^{15}$ However, Shaikh's study population was small and not powered to detect a true difference in endophthalmitis rates following the two surgical techniques.

Eifrig et $\mathrm{l}^{12}$ followed 15326 cases of 20-gauge vitrectomy and found the rate of endophthalmitis to be $0.039 \%$. This greater incidence may be due to postoperative endophthalmitis being defined as acute infection within 6 weeks of surgery, whereas the current study used the criterion of 14 days after surgery. In addition, they included records dating from as early as 1984, and since then there have been several advances in 20-gauge surgery that may have resulted in decreased rates of endophthalmitis.

The current study's incidence of endophthalmitis after 25 -gauge vitrectomy $(0.23 \%)$ was similar to the rate of 
$0.23 \%$ that Kunimoto and Kaiser reported. ${ }^{18}$ Although these findings were not statistically significant due to the low number of measured outcomes, they represent a sevenfold higher incidence of endophthalmitis with 25 -gauge vitrectomy compared with 20-gauge vitrectomy. The trend is worrisome and consistent with the two previous reports by Kunimoto and Kaiser ${ }^{18}$ and Scott. ${ }^{15}$

It is possible that the reported case of endophthalmitis after combined 25-gauge vitrectomy and cataract surgery occurred secondary to the cataract surgery rather than the vitrectomy portion of the procedure even though the corneal incision was sutured and did not leak. Cataract surgery is associated with a higher risk of postoperative endophthalmitis than is vitrectomy, with cited rates ranging from 0.1 to $0.2 \% .^{20-22}$ Alternatively, combining the surgeries may have increased the risk of endophthalmitis. The data did not show any increased risk when combination surgery was conducted using 20-gauge vitrectomy. As cataract progression may occur in up to $80 \%$ of patients after vitrectomy, the combination procedure seems a logical choice in patients with visually significant cataract and vitreoretinal pathology. Although phacoemulsification has often been performed with 20-gauge surgery, ${ }^{23}$ there is limited evidence addressing the safety of 25-gauge vitrectomy in combination with cataract surgery. Use of a clear corneal incision, as in case 1 , has been recommended for cataract surgery to minimize disruption of conjunctival protection of sclerotomy site following sutureless, small-incision vitrectomy. Oshima et $a l^{10}$ retrospectively evaluated 144 patients who underwent combination 25-gauge vitrectomy and clear corneal phacoemulsification, and found that postoperative complications included 18 cases of hypotony, 1 case of retinal detachment, and no cases of endophthalmitis. Chang et $a l^{23}$ also compared outcomes in 15 eyes undergoing 20-gauge and 15 eyes undergoing 25-gauge vitrectomy combined with clear corneal phacoemulsification. They reported that combined sutureless surgery with 25-gauge vitrectomy needed shorter set-up time and caused less postoperative ocular irritation than combined surgery with 20-gauge vitrectomy, and no cases of endophthalmitis were reported in either group. In addition, Huang et al ${ }^{24}$ examined 20 patients undergoing combined 25-gauge vitrectomy and clear corneal phacoemulsification and found complications to include posterior capsule tear, partial ciliary body detachment, and hyphaema. None of these studies, however, were adequately powered to assess an increased risk for endophthalmitis.

Several methods have been proposed to potentially decrease the risk of endophthalmitis following transconjunctival sutureless surgery, such as use of angled or bevelled incisions, misalignment of conjunctival and scleral wounds to avoid a continuous tract for bacterial influx, suture placement when bleb formation secondary to leakage at the sclerotomy site is seen upon cannula removal, performing intraocular fluid-air/gas exchange at the completion of surgery, and fluid infusion when a shallow chamber is seen upon cannula removal. ${ }^{2,4}$ It is difficult to assess the success of these modifications, as these theoretical improvements have been largely documented in smaller scale studies inadequately powered to find such a rare complication as endophthalmitis. ${ }^{4,6}$ Kunimoto and Kaiser reported seven endophthalmitis cases, in all of which the technique of displacing the conjunctiva for 25-gauge trocar and cannula placement was used but did not prevent endophthalmitis. ${ }^{18}$ In addition, Kunimoto and Kaiser postulated that a gas fill may improve wound integrity at the conclusion of 25-gauge vitrectomy and prevent endophthalmitis. However, Shaikh reported an increased rate of endophthalmitis $(1.6 \%)$ in 129 cases despite the use of a partial gas fill and bevelled incisions. ${ }^{19}$ Lastly, it has been often speculated that unsutured wounds make postoperative hypotony a risk factor for endophthalmitis. Interestingly, there was no hypotony on postoperative day 1 in the one case in our series, the seven from Kunimoto and Kaiser, and the three individual case reports of endophthalmitis following 25-gauge vitrectomy. ${ }^{11,13,14,18}$ In the 11 cases reported by Scott et al, the median IOP was $13 \mathrm{~mm} \mathrm{Hg}$ (range: $5-27 \mathrm{~mm} \mathrm{Hg}$ ). Should additional studies confirm an increased risk of endophthalmitis following 25-gauge vitrectomy, further research will be required to determine which surgical modifications will prevent endophthalmitis in 25-gauge vitrectomy.

This study has several limitations. The sample size did not yield enough cases of endophthalmitis to gain statistical significance. In addition, 20-gauge vitrectomy and 25-gauge vitrectomy are performed in different patient populations, introducing possible selection bias. The 25-gauge system is typically indicated for cases that do not require extensive peripheral membrane dissection. ${ }^{2}$ However, case selection bias reflects standard practice and likely will remain representative of expected rates of complication following 25-gauge vitrectomy. Furthermore, this study was limited by its retrospective nature. Although it is possible that not all cases of endophthalmitis were identified, retina surgeons involved in this study follow patients in the acute postoperative period so it is unlikely that acute infections (within 14 days of surgery) were missed. However, billing codes were used to identify potential cases, so it is possible that some cases could have been missed using this methodology.

In summary, this study suggests that the incidence of acute postoperative endophthalmitis is low in both 
25- and 20-gauge vitrectomy, although there may be an increased risk following 25 -gauge vitrectomy in comparison to 20-gauge vitrectomy. However, this observed difference was not statistically significant given the low number of measured outcomes. This trend is consistent with recent published studies and raises a possible concern regarding 25-gauge vitrectomy.

However, retrospective studies have inherent biases and may not accurately reflect the true incidence of endophthalmitis after sutureless 25 -gauge vitrectomy. A prospective, randomized multi-centre study is necessary to address this issue, but would require tens of thousands of participants to achieve statistical power given the low incidence rates.

\section{References}

1 Fujii GY, De Juan Jr E, Humayun MS, Pieramici DJ, Chang $\mathrm{TS}$, Awh C et al. A new 25-gauge instrument system for transconjunctival sutureless vitrectomy surgery. Ophthalmology 2002; 110: 1807-1812.

2 Chen E. 25-gauge transconjunctival sutureless vitrectomy. Curr Opin Ophthalmol 2007; 18: 188-193.

3 Fujii GY, De Juan Jr E, Humayun MS, Chang TS, Pieramici DJ, Barnes A et al. Initial experience using the transconjunctival sutureless vitrectomy system for vitreoretinal surgery. Ophthalmology 2002; 109: 1814-1820.

4 Lakhanpal RR, Humayun MS, de Juan Jr E, Lim JI, Chong LP, Chang TS et al. Outcomes of 140 consecutive cases of 25-gauge transconjunctival surgery for posterior segment disease. Ophthalmology 2005; 112: 817-824.

5 Kellner L, Wimpissinger B, Stolba U, Brannath W, Binder S. 25-gauge vs 20-gauge system for pars plana vitrectomy: a prospective randomized clinical trial. Br J Ophthalmol 2007; 91: 945-948.

6 Rizzo S, Genovesi-Ebert F, Murri S, Belting C, Vento A, Cresti $\mathrm{F}$ et al. 25-gauge, sutureless vitrectomy and standard 20-gauge pars plana vitrectomy in idiopathic epiretinal membrane surgery: a comparative pilot study. Graefes Arch Clin Exp Ophthalmol 2006; 244: 472-479.

7 Cho YJ, Lee JM, Kim SS. Vitreoretinal surgery using transconjunctival sutureless vitrectomy. Yonsei Med J 2004; 45: 615-620.

8 Okuda T, Nishimura A, Kobayashi A, Sugiyama K. Postoperative retinal break after 25-gauge transconjunctival sutureless vitrectomy: report of four cases. Graefes Arch Clin Exp Ophthalmol 2007; 245: 155-157.

9 Ibarra MS, Hermel M, Prenner JL, Hassan TS. Longer-term outcomes of transconjunctival sutureless 25-gauge vitrectomy. Am J Ophthalmol 2005; 139: 831-836.

10 Oshima Y, Ohji M, Tano Y. Surgical outcomes of 25-gauge transconjunctival vitrectomy combined with cataract surgery for vitreoretinal diseases. Ann Acad Med Singapore 2006; 35: 175-180.

11 Taylor SR, Aylward GW. Endophthalmitis following 25-gauge vitrectomy. Eye 2005; 19: 1228-1229.

12 Eifrig CW, Scott IU, Flynn Jr HW, Smiddy WE, Newton J. Endophthalmitis after pars plana vitrectomy: incidence, causative organisms, and visual acuity outcomes. Am J Ophthalmol 2004; 138: 799-802.

13 Taban M, Ufret-Vincenty RL, Sears JE. Endophthalmitis after 25-gauge transconjunctival sutureless vitrectomy. Retina 2006; 26: 830-831.

14 Acar N, Unver YB, Altan T, Kapran Z. Acute endophthalmitis after 25-gauge sutureless vitrectomy. Int Ophthalmol 2007; 27: 361-363.

15 Scott IU, Flynn Jr HW, Dev S, Shaikh S, Mittra RA, Arevalo JF et al. Endophthalmitis after 25-gauge and 20-gauge pars plana vitrectomy. Retina 2008; 28: 138-142.

16 Cohen SM, Flynn Jr HW, Murray TG, Smiddy WE. Endophthalmitis after pars plana vitrectomy. The Postvitrectomy Endophthalmitis Study Group. Ophthalmology 1995; 102: 705-712.

17 Taban M, Sarayba MA, Ignacio TS, Behrens A, McDonnell PJ. Ingress of India ink into the anterior chamber through sutureless clear corneal cataract wounds. Arch Ophthalmol 2005; 123: 643-648.

18 Kunimoto DY, Kaiser RS. Incidence of endophthalmitis after 20- and 25-gauge vitrectomy. Ophthalmology 2007; 114: 2133-2137.

19 Shaikh S, Ho S, Richmond PP, Olson JC, Barnes CD. Untoward outcomes in 25-gauge vs 20-gauge vitreoretinal surgery. Retina 2007; 27: 1048-1053.

20 Javitt JC, Street DA, Tielsch JM, Wang Q, Kolb MM, Schien $\mathrm{O}$ et al. National outcomes of cataract extraction. Retinal detachment and endophthalmitis after outpatient cataract surgery. Cataract Patient Outcomes Research Team. Ophthalmology 1994; 101: 100-105; discussion 106.

21 Kattan HM, Flynn Jr HW, Pflugfelder SC, Robertson C, Forster RK. Nosocomial endophthalmitis survey. Current incidence of infection after intraocular surgery. Ophthalmology 1991; 98: 227-238.

22 Aaberg Jr TM, Flynn Jr HW, Schiffman J, Newton J. Nosocomial acute-onset postoperative endophthalmitis survey. A 10-year review of incidence and outcomes. Ophthalmology 1998; 105: 1004-1010.

23 Chang CJ, Chang YH, Chiang SY, Lin LT. Comparison of clear corneal phacoemulsification combined with 25-gauge transconjunctival sutureless vitrectomy and standard 20-gauge vitrectomy for patients with cataract and vitreoretinal diseases. J Cataract Refract Surg 2005; 31: 1198-1207.

24 Hwang JU, Yoon YH, Kim DS, Kim JG. Combined phacoemulsification, foldable intraocular lens implantation, and 25-gauge transconjunctival sutureless vitrectomy. J Cataract Refract Surg 2006; 32: 727-731. 